\title{
ANÁLISIS DE LA COBERTURA DEL HOMICIDIO, DESDE EL ENFOQUE DE PRODUCCIÓN NOTICIOSA, EN EL DIARIO "AL DÍA”
}

\author{
ANALYSIS OF THE COVERAGE OF THE HOMICIDE, FROM THE \\ PERSPECTIVE OF NEWS PRODUCTION, THE TABLOID "AL DÍA"
}

\author{
Isaías Molina Jácome ${ }^{1}$. Universidad Autónoma del Caribe. Colombia.
}

\section{RESUMEN}

Este artículo analiza la cobertura de las noticias sobre homicidios, en la etapa de su producción, enfocándose en los elementos con los que el diario sensacionalista 'Al Día' (Barranquilla-Colombia) da relevancia a las noticias y en los valores usados por los reporteros para elaborar la noticia. Para el análisis se utilizaron los estudios de la Sociología de la Producción Noticiosa o Newsmaking, como base teórica, con una metodología de corte cuali-cuantitativo, que cruzó un análisis de contenidos de 240 noticias de homicidio sucedidos durante cerca de cinco años (2008 a mayo 2012), y 6 entrevistas en profundidad realizadas con el equipo de periodistas. Se halló que el tema más importante en la cobertura de noticias es el hecho de sangre más reciente, que el periodista usa una combinación de fuentes que incluye: familiares, testigos, autoridades y vecinos. Se identifican tres valores noticiosos: interés, cercanía y repercusión en la sociedad. Se concluye que las autoridades no tienen el monopolio de la información en el caso de los homicidios y que los valores noticiosos tradicionales se aplican también a este medio sensacionalista, pero que, existen otros como: impacto, cercanía y repercusión.

PALABRAS CLAVE: medios masivos, noticias, cobertura, homicidios, Sociología, producción noticiosa, Newmaking.

\section{ABSTRACT}

This article analyzes the news coverage of homicide in the stage production focusing on the elements with which the tabloid 'Al Día' (Barranquilla-Colombia) gives prominence to news, and in the values used by reporters to elaborate news. The analysis used as a theoretical basis studies is the sociology of news production or Newsmaking, with a methodology of qualitative and quantitative nature, which crossed a content analysis of 240 news about homicides happened in five years (2008 to May 2012), and six interviews in depth with the team of journalists. It was found that the most important news coverage issue is the fact that more fresh blood, the journalist uses a combination of sources including: relatives, witnesses, authorities and neighbors. Three news values are identified:interest, proximity and impact on society. It is concluded that the authorities do not have the monopoly on information in the case of the murders and that traditional news values also apply to the tabloid media, but there are others such as impact, proximity and impact.

\footnotetext{
${ }^{1}$ Isaías Molina Jácome: Universidad Autónoma del Caribe. Barranquilla, Colombia.
} 
KEY WORDS: Mass media, newscoverage, homicides, Sociology, Newsmaking.

\section{Cómo citar el artículo:}

Molina Jácome, I. (2017). Análisis de la cobertura del homicidio, desde el enfoque de producción noticiosa, en el diario "Al día". Revista de Ciencias de la Comunicación e Información, 22(2), 37-50.

doi: http://doi.org/10.35742/rcci.2017.22(2).37-50

\section{INTRODUCCIÓN}

El diario 'Al Día' es un medio gráfico sensacionalista caracterizado por contar historias sobre crímenes o delitos que se generan en la ciudad de Barranquilla (Colombia). Las historias producidas por los reporteros con una mayor o menor cobertura aparecen en las primeras siete páginas del diario, en una sección denominada "Así fue", que incluye temas como: homicidios o crímenes, robos, accidentes, denuncias ciudadanas, suicidios, riñas y capturas de la policía.

La producción noticiosa del diario 'Al Día' es consumida por los sectores populares de la ciudad donde circula y en la que habita más del $50 \%$ de la población, que en la actualidad alcanza el millón y medio de habitantes, según el último censo de Barranquilla 2005, realizado por el Departamento Administrativo Nacional de Estadísticas, DANE. Estos elementos son suficientes para generar el interés de investigar sobre qué sucede en la cobertura de noticias de este medio y cómo los periodistas llevan a cabo la producción de cada noticia que circula a diario.

Para analizar la producción noticiosa se usó el enfoque de la Sociología de las noticias o estudios del Newsmaking, puesto que el interés era no solo entender cómo se da la cobertura de las noticias, sino también las rutinas periodísticas que intervienen en la producción de los mensajes. Algunas de las preguntas que motivaron el análisis fueron: ¿Qué estrategias utilizan los medios en su cobertura para darle importancia o relevancia a las noticias?, ¿Cómo miden los periodistas el grado de interés de la noticia? y ¿Cómo los periodistas establecen, dentro de su rutina diaria, la mayor o menor cobertura de una noticia? Los hallazgos sobre cómo establece el diario la importancia de las noticias sobre homicidios y los valores que los periodistas utilizan para medir el interés de los lectores se obtuvieron aplicando la metodología del análisis de contenido y entrevistas en profundidad. En el siguiente aparte se hace un recuento de la cobertura de noticias y la instancia de producción noticiosa con los autores más citados de la literatura académica.

Los hallazgos sobre cómo establece el diario la importancia de las noticias sobre homicidios y los valores que los periodistas utilizan para medir el interés de los lectores se obtuvieron aplicando la metodología del análisis de contenido y entrevistas en profundidad. En el siguiente aparte se hace un recuento de la cobertura de noticias y la instancia de producción noticiosa con los autores más citados de la literatura académica. 


\section{ANÁLISIS DE LA COBERTURA DEL HOMICIDIO, DESDE EL ENFOQUE DE PRODUCCIÓN NOTICIOSA, EN EL DIARIO "AL DÍA"}

\subsection{La cobertura de noticias y la instancia de producción}

Para analizar la cobertura del homicidio es necesario reconstruir la trayectoria de los trabajos más representativos de la cobertura de noticias y la instancia de producción del mensaje. Al revisar la literatura académica se encuentra el enfoque de Tuchman (1973), una de las autoras pioneras que han ayudado a consolidar la investigación en instancia de producción de la noticia y cuyo foco de interés descansa en cómo una organización mediática puede procesar los eventos inesperados o cómo los periodistas disminuyen la variabilidad de los eventos que forman la materia prima de la noticia. Tuchman (1973) abordó esas preguntas a través de la observación directa en las salas de redacción de los medios y mediante el acompañamiento a los periodistas a cubrir sus historias. Su texto "Making News by Doing Work: Routinizing the Unexpected", es una de las primeras referencias de la producción de las noticias como objeto de estudio.

Otro de los ejemplos sobre cómo los investigadores han abordado los problemas de la producción de la noticia es el texto de Lance, Gressett y Haltom (1985), donde reflexionan sobre lo que es o no es noticia en el marco de una reparación noticiosa o rectificación o el de Randall, Lee-Sammons y Hagner (1988), quienes en su estudio sobre cobertura de las noticias de delincuencia común y el crimen de élite, plantean que los periodistas enfatizan la etapa más dramática y temprana del proceso judicial, además que los medios le prestan una mayor atención a la delincuencia común que a otras noticias y que existe una identidad básica en la forma en que los diferentes medios las presentan. Por su parte, Schudson (1989), revisa el campo académico denominado Sociología de la producción noticiosa y es, según Hernández (1997), uno de los referentes en esta área de estudio que tiene diversas denominaciones, a saber: Sociología de las noticias, Sociología del periodismo, Sociología de la producción noticiosa, Fabricación de la noticia, Construcción de la noticia o del acontecimiento, Estudios del Newsmaking,Sociología de los periodistas o Sociología de las organizaciones de noticias. Asimismo, Hernández (1997), al revisar la literatura identifica la necesidad de que la Sociología de la producción de noticias, aborde tres aspectos fundamentales: el conocimiento de la práctica periodística, la enseñanza del periodismo y el establecimiento de relaciones de la investigación con los medios.

Aparte de Tuchman (1973), Schudson (1989) y Hernández (1997) existen autores como Rodrigo (1989) y Fontcuberta (1993), quienes abordan la noticia desde su construcción. Rodrigo (1989) plantea que el mensaje de los textos periodísticos es fabricado a diario por los periodistas en un ciclo de producción, circulación y reconocimiento y Fontcuberta (1993), considera que si antes eran los historiadores quienes evaluaban 'qué era' y 'qué no era' acontecimiento histórico, ahora son los medios quienes cumplen esa función.

De acuerdo a Fontcuberta (1993), la producción de la noticia comienza con el acontecimiento, que es una ruptura con la cotidianidad y es el resultado de un hecho trascendente, el cual depende tanto del protagonista y de las circunstancias como de su desarrollo posterior. Aparte de eso, Canel y Sánchez-Aranda (1999) reconocen otros dos factores del campo teórico que son: las actitudes profesionales que influyen en las noticias y los elementos organizacionales (el tipo de medio, la propiedad o el entorno de redacción), que para ellos son los elementos que 
configuran más las actitudes profesionales, y según Reese y Ballinger (2001), le introducen un sesgo a la información con su subjetividad y promueven el control social desde los medios.

A los anteriores factores de la instancia de producción -tanto el periodista como la organización en la cual trabaja-, Feinberg (2002), agrega el hecho de que los periodistas contribuyen a construir una imagen eficaz de las acciones de la policía y esto ocurre, porque las historias relacionadas con el crimen, donde interviene la policía como una fuente de información, ocupan un lugar destacado en las páginas, es decir, se les da mayor importancia en la cobertura de las noticias como lo evidencia en su estudio sobre la influencia de la cobertura de un periódico local sobre el tamaño de la policía municipal-en 63 grandes ciudades norteamericanas-; donde tuvo en cuenta como variables independientes: la cobertura de la prensa local, las estadísticas de crímenes violentos, la población minoritaria, la pobreza, la desigualdad de los ingresos, la organización del gobierno local y los cambios en el tamaño de los departamentos de policía.

Por otro lado, el hecho de que las noticias que relatan el crimen, en especial las que cuentan los asesinatos, tienen una alta presencia o 'noticiabilidad' de larga data en los medios, lo confirma Lundman (2003), quien en sus hallazgos muestra que las historias que relatan el crimen o los asesinatos son producto de las evaluaciones periodísticas tanto en raza como en género. Es decir, que en el caso de un diario, para decidir si se debe cubrir o no un asesinato, la cantidad de cobertura que tendrá $y$, donde se ubicarán las historias, los periodistas tienen en cuenta los estereotipos de raza y género que predominan en su cultura.

El estudio tuvo en cuenta alrededor de 640 homicidios de 1984 a 1992, de asesinatos con victimario conocido y víctimas de raza y género. Además, plantea la necesidad de investigar más sobre el efecto de la exposición a los medios, con respecto a las noticias de homicidio, teniendo en cuenta las actitudes hacia la raza blanca, las creencias sobre los afroamericanos y las actitudes y creencias masculinas sobre las mujeres.

Desde el punto de vista de las fuentes Carpenter, Lacy y Fico (2006), en su estudio sobre la cobertura noticiosa de los crímenes de alto perfil reporta en sus hallazgos que las historias que involucran crímenes de este tipo tienen fuentes más transparentes en comparación con otras historias. Pero esto no significa que exista una pluralidad de fuentes, pues al contrario de lo que podría pensarse, según este estudio, esas historias fueron más propensas a ser contadas desde una sola perspectiva noticiosa y a contener fuentes anónimas. La investigación examinó el uso de las fuentes y los esfuerzos de los periodistas para proporcionar un contexto para este tipo de crímenes en comparación con la cobertura de otras historias con una cobertura fuerte, que ocurrieron en el año 2004, en medios como: World News Tonight (Cadena $A B C$ ), Evening News (Cadena CBS) y Nightly News (Cadena $N B C)$. Los noticieros de la mañana fueron: Good Morning America $(A B C)$, The Early Show (CBS), y The Today Show (NBC).

Con respecto a los estereotipos mencionados como es el caso de las nacionalidades, Lasorsa y Jia (2007), en su estudio "Cuando los periodistas 


\section{ANÁLISIS DE LA COBERTURA DEL HOMICIDIO, DESDE EL ENFOQUE DE PRODUCCIÓN NOTICIOSA, EN EL DIARIO "AL DÍA"}

engañan", sobre la producción de estereotipos en los periodistas, plantean en sus hallazgos la existencia de la teoría de la automaticidad de los estereotipos en los medios, a menos que un receptor tenga la capacidad de no dejarse influir por el estereotipo. El estudio analizó 183 historias publicadas por diez periodistas, que resultaron ser engañosas teniendo en cuenta variables como: ocupación, nacionalidad, región dentro de una nación, clase social, raza y religión. Sin embargo, desde otro enfoque, Marchetti (2008), con respecto a la cobertura mediática y la instancia de producción propone lo que él denomina "Teoría de los campos", que consiste en integrar campos como el económico o político con el campo periodístico. De acuerdo a esto, el campo periodístico se ha caracterizado a lo largo de la historia por representar una oposición entre dos polos.

El primero busca desde una lógica comercial atraer al mayor número de lectores y el segundo, de difusión más restringida, está dirigido a públicos a menudo más segmentados. Según él, este modelo podría representarle dos ventajas al investigador: primero, distinguir niveles en el análisis del campo profesional del periodismo y segundo, reconocer las condiciones económicas, políticas, discursivas, profesionales y sociales que determinan el trabajo periodístico. Asimismo, este autor evidencia la escasez de estudios de la Sociología de los medios con respecto, a las condiciones de producción de la información y a los estudios del discurso mediático.

Cabe destacar también los estudios de Aguilón (2008) sobre la cobertura mediática del suceso noticioso, donde plantea que el diseño gráfico de los periódicos resaltó la espectacularidad de la noticia y más adelante Aguillón (2010), en su investigación sobre cómo la prensa escrita venezolana construye la ideología en los textos noticiosos referidos a la violencia delictiva, señala que, "a pesar de su irrelevancia pública, los familiares de las víctimas y testigos se convierten en las voces informativas más privilegiadas en los turnos de palabras que los periodistas otorgan en los textos" (2010, p. 98).

Por ello, es que las rutinas laborales de los periodistas es otro de los aspectos relacionados con la cobertura mediática y la instancia de producción, al respecto Frankenberg y Lozano-Rendón (2010) Ilevaron a cabo un estudio con cuatro directores de medios informativos en México, donde usaron la metodología de entrevistas en profundidad. En sus hallazgos identificaron las rutinas de trabajo y las influencias individuales, que moldean e inciden en los contenidos noticiosos con variables como: sexo, edad, clase social, religión, ideología política, que evidencian la relevancia que tienen en la construcción de la noticia y en los enfoques utilizados por el medio.

Complementando lo anterior, en la revisión de la literatura, el estudio de Morales y Vallejo (2011) acerca de los "Estándares de calidad en la información sobre seguridad en Bogotá en medios nacionales y locales" halló que, en las noticias de la muestra, se da una dependencia de las fuentes institucionales y oficiales, y un abuso de las fuentes personales (para subrayar los efectos dramáticos de la información), que son los factores que hablan de la calidad de la producción periodística. En esta investigación se usó una metodología de análisis de contenidos, con el método de muestreo de semana compuesta, en un periodo de seis meses, con un corpus de 1.276 piezas, entre informativas y de opinión. 
Se tuvieron en cuenta cuatro periódicos: El Tiempo, El Espectador, El Espacio y Hoy; dos noticieros de la noche: Canal Capital y City TV; y emisiones del medio día en cuatro emisoras: Alerta Bogotá, RCN, Caracol y Radio Santa Fe. Además, entrevistas en profundidad con 28 reporteros y editores del área judicial, de 10 medios de comunicación de los ámbitos local y nacional.

\section{OBJETIVOS}

Este artículo tuvo como objetivo analizar la cobertura del homicidio en la instancia de producción de la noticia, a partir de dos factores: el primero, los elementos con que el diario sensacionalista 'Al Día' da relevancia a las noticias de delito y, el segundo, los valores que los periodistas utilizan para la cobertura noticiosa.

\section{METODOLOGÍA}

El análisis de contenidos utilizó dos instrumentos: una ficha de análisis y un libro de códigos. El instrumento se adaptó de la tesis doctoral de Muñiz (2007) denominada "Encuadres noticiosos e inmigración: del análisis de los contenidos al estudio de los efectos mediáticos". Además del análisis de contenidos se incluyó una instancia donde se realizaron entrevistas estructuradas al editor, al jefe de redacción, coordinador de judiciales y a los redactores del medio estudiado, usadas para obtener datos de la cobertura noticiosa por parte de los periodistas.

Universo de análisis: se incluyen todas las noticias desde 2007, año en que inició la circulación del diario 'Al Día', hasta el año 2012. Muestra: se estudiaron casi cinco años de circulación que comprenden: 2008, 2009, 2010, 2011 y mayo de 2012. Para la selección se usó la técnica de muestreo de semana compuesta. Cuando la semana no tenía los días seleccionados se determinó escoger los del día anterior o posterior (Kayser, 1982).

Codificación y fiabilidad: se conformó un equipo de codificadores a quienes se les entrenó en el uso del instrumento cuantitativo (Ficha de análisis y libro de códigos), para luego proceder a llevar a cabo el análisis de contenido. Además, se practicó una fiabilidad intercodificadores tal como lo expone Igartúa (2006, p. 218), quien manifiesta que si la codificación no es fiable el análisis carece de confianza, por lo cual se convierte en un requisito imprescindible en todo análisis de contenido, cuya información debe incluirse en todo trabajo de investigación de calidad y más aún si se pretende publicar en una revista científica. La ventaja de la fiabilidad intercodificadores es que permite dividir el trabajo de codificación entre diferentes analistas.

El procedimiento consistió en recodificar las variables en un $20 \%$ de la muestra para evitar sesgos entre los distintos codificadores del instrumento. La selección se hizo en forma aleatoria y esta instancia fue realizada por los mismos codificadores que trabajaron sobre la muestra. Esta fase tuvo dos etapas una que se hizo en la Fase de entrenamiento de los codificadores (fase de pilotaje) y al finalizar el estudio, tal como lo plantea Igartúa (2006). Por otro lado, el nivel de acuerdo mínimo que se debe alcanzar para los datos sean fiables es de $0.80 \%$ (80\%). 


\section{ANÁLISIS DE LA COBERTURA DEL HOMICIDIO, DESDE EL ENFOQUE DE PRODUCCIÓN NOTICIOSA, EN EL DIARIO "AL DÍA"}

\section{RESULTADOS}

Con respecto al objetivo de describir cómo se establece la importancia de las noticias sobre homicidios, publicadas en la sección "Así fue", del diario 'Al Día', que circula en Barranquilla (Colombia); podemos mencionar que ésta aparece en portada en un $75,0 \%$ de las noticias de la muestra estudiada y abre la sección "Así fue" en solo un $31,7 \%$, es decir, que la noticia de homicidio casi nunca abre la sección en un $68,3 \%$ de las noticias y tampoco aparece en páginas impares-que son las que más se ven-, puesto que la mayoría de las veces, es decir, en un $57,1 \%$, la página en la que empieza es par. Esto es explicado por la publicidad que contiene el medio y que traslada la noticia principal a la página número 4 , cuando debería ser en la página 3 , es decir, que cuando esto ocurre no existen avisos publicitarios y las noticias del delito comienzan en página impar. Por otro lado, en cuanto a la variable tamaño de la noticia los dos tamaños que más predominan son: el que tiene entre dos y tres cuartos de página, donde se nota la presencia de las noticias de homicidios en un $34,6 \%$ y el que tiene dimensiones entre uno y dos cuartos de página, donde las noticias de homicidios alcanzan un $20,8 \%$ del tamaño.

En cuanto a las estrategias de usar elementos gráficos distintos al texto escrito, estos están presentes en un $98,3 \%$ de las noticias de la muestra, con fotografías en un $97,9 \%$ de ellas. Al indagar si existían gráficos y esquemas se descubrió que nunca se presentan en un $100 \%$ de los casos y lo mismo ocurre con las infografías. Elementos gráficos como los recuadros aparecen utilizados en un $58,8 \%$ de las noticias y en cuanto al número de fotografías, que es una de las variables que mejor ayudarían a entender la importancia que el medio da a las noticias de homicidio, se encontró que siempre hay más de una, en un $61,3 \%$ de la muestra y en el caso de que solo se haya utilizado una este porcentaje representa un $36,7 \%$ de los casos estudiados.

En la muestra cuando la noticia utiliza una sola fuente el $29,6 \%$ proviene de familiares, el $20,8 \%$ de testigos y el $17,1 \%$ de autoridades y un $10,4 \%$ de vecinos. En suma, alrededor de un $77,9 \%$ de la información que usa el periodista en la cobertura de la noticia proviene de fuentes como: familiares, testigos, autoridades y vecinos. Por otro lado, cuando la noticia utiliza dos fuentes el $20,8 \%$ proviene de amigos y familiares, el $11,3 \%$ proviene de vecinos y autoridades y un $9,2 \%$ proviene de vecinos y familiares. En total, en el caso del uso de dos fuentes en la muestra de noticias el $41,3 \%$ proviene de amigos y familiares, vecinos y autoridades y vecinos $\mathrm{y}$ familiares. $Y$ cuando la noticia utiliza tres fuentes el $12,5 \%$ proviene de vecinos, amigos y familiares, y testigos. En resumen, los hallazgos obtenidos al estudiar el comportamiento de las fuentes en las noticias de la muestra arrojaron lo siguiente: Cuando en las noticias se usa una sola fuente la mayor parte de la información la proveen los familiares, en un 29,6\%; cuando en la noticia aparecen dos fuentes, la mayor parte de la información la suministran los amigos y familiares y esto representa un 20,8\%; y cuando están presentes tres tipos de fuentes en las noticias de la muestra la mayor parte de la información proviene de los vecinos, y esto alcanza un $5,8 \%$. La suma de los porcentajes anteriores equivale a un $56,2 \%$, que representa la mayoría de las fuentes presentes en la muestra de las 240 noticias estudiadas. La tabla 1 que aparece a continuación tiene la intención de resumir los hallazgos cuantitativos, que se obtuvieron al intentar establecer cómo el diario 'Al 
Día' logra darle importancia a las noticias del delito que expone en la sección "Así fue".

Tabla 1. Cómo se establece la importancia de las noticias.

\begin{tabular}{|l|c|}
\hline \multicolumn{1}{|c|}{ NOMBRE DE LA VARIABLE } & PORCENTAJE \\
\hline Aparece la noticia en portada & \begin{tabular}{c}
$75,0 \%$ \\
\hline Abre la sección
\end{tabular} \\
\hline Página en la que empieza $68,3 \%$ \\
\hline Tamaño de la noticia & $\begin{array}{r}\text { Par en un } 57,1 \% \\
\text { Elementos gráficos } \\
\text { uno y dos cuartos de página: } 34,6 \% \text { y entre }\end{array}$ \\
\hline Fotografía & Si en un $98,3 \%$ \\
\hline Recuadro & Si en un $97,9 \%$ \\
\hline Gráficos y esquemas & Si en un $58,8 \%$ \\
\hline Infografías & No se presentan en un $100 \%$ \\
\hline Número de fotografías & No se presentan en un $99,2 \%$ \\
\hline Fuentes & $\begin{array}{r}\text { Más de una en un } 61,3 \% \text { y solo una en un } 36,7 \% \\
\text { de los casos estudiados. }\end{array}$ \\
\hline
\end{tabular}

Por otra parte, en la instancia cualitativa se halló que los criterios de selección que los reporteros del diario 'Al Día' usan al momento de producir la noticia deben contener tres elementos:

1. Impacto.

2. Cercanía.

3. Repercusión en la sociedad.

Esto lo expresan los periodistas en distintos apartes de las entrevistas, a través de las cuales se profundizaron los hallazgos cuantitativos del análisis de contenidos:

- "Una noticia de impacto, una noticia de cercanía, una noticia que tenga mucha repercusión en la sociedad, que tenga importancia", (Roberto Llanos, Director del diario 'Al Día').

- "Cualquier hecho que ocurra en vía pública en Barranquilla, el cual nosotros consideremos que sea de interés periodístico, (Wilson Rodríguez, Coordinador de judiciales del diario 'Al Día').

- "La imagen y el impacto que tienen en la sociedad, por ejemplo en el periódico de hoy podemos ver que estamos dándole prelación en la portada, a una racha criminal que tiene atemorizada a la ciudad y a su área metropolitana de 4 homicidios en 4 horas, esto sin duda que genera impacto en la comunidad porque hay zozobra de la gente", (Jimmy Cuadros, Jefe de redacción del diario 'Al Día').

- "Actualidad... depende la sección... el muerto más reciente o el hecho de sangre más reciente, muerto herido, un hecho, de crónica judicial", (Andrés Gaitán, periodista).

En la instancia cualitativa también se halló que los periodistas reconocen que la portada si bien es una estrategia para darle importancia a la noticia, a veces los temas judiciales le dan paso a las noticias deportivas, porque estas captan la 


\section{ANÁLISIS DE LA COBERTURA DEL HOMICIDIO, DESDE EL ENFOQUE DE PRODUCCIÓN NOTICIOSA, EN EL DIARIO "AL DÍA"}

atención del lector. "El $98 \%$ de nuestras portadas siempre son temas judiciales, algunas ocasiones hemos usado la primera plana para resaltar temas deportivos, bien sea títulos del Junior (el equipo de fútbol local), selección Colombia, eventos deportivos y acontecimientos deportivos que generen 'lecturabilidad', que generen atención en la gente", (Jimmy Cuadros, Jefe de redacción). Además, que existe un uso casi exclusivo de la fotografía como recurso gráfico en el diario y que los periodistas tienen una buena relación con sus fuentes, lo cual les permite tener un acercamiento con sus lectores, pero que esta relación no es fácil, pues cubrir un asesinato los involucra en el momento doloroso por el que están pasando los familiares o seres queridos de la víctima y esto no solo los afecta, sino que les impide llevar a cabo su trabajo.

"Muchos familiares no están en condiciones o no quieren hablar, se reúsan e incluso te pueden amenazar, te increpan o te gritan, algunas veces te intentan golpear y uno entiende eso porque están dolidos, el trabajo de nosotros tampoco es fácil, no es fácil porque no sé, bueno no sé para quien sería fácil acercársele a alguien al que le acaban de matar un familiar a preguntarle datos sobre esa persona si esa persona en ese momento está llena de dolor, de ira, de rabia, de todo", Jimmy Cuadros, (Jefe de redacción).

"Aunque uno ya tenga 20 años de experiencia en esto, el hecho de llegar a cubrir una noticia donde haya un muerto, el ver un cadáver ahí tirado eso golpea, eso pega, eso a nadie... nadie se vuelve insensible a una tragedia, y mucho más cuando se trata de una mujer o hay niños", (Wilson Rodríguez, Coordinador de judiciales).

"Si en tu escenario ocurre un hecho judicial, un homicidio, tú quieres saber quién fue al que asesinaron, cómo quedó esa persona, porque muchas veces tu no vas al lugar de los hechos porque te da miedo; hay un morbo, un morbo en la gente de ver al muerto, digamos que en ese sentido siempre le vamos a dar prelación a la foto de un homicidio que tenga un cadáver", (Jimmy Cuadros, Jefe de redacción).

Pese a ello, a los periodistas se les exige utilizar el mayor número de fuentes posibles, es decir, ser 'pluri-fuentistas'. Así lo muestran las siguientes citas producto de las entrevistas realizadas a los periodistas:

"Por eso tenemos buenas fuentes, hay buenas relaciones con las fuentes que cubrimos y eso nos permite tener un acercamiento, un detalle más de lo que está pasando y que la comunidad no conoce o no tiene acceso a ella", (Diana Ospino, periodista).

"El $70 \%$ de nuestras informaciones son suministradas por la comunidad. Los temas que manejamos los domingos son temas que la gente llama aquí, tenemos una secretaria que los recibe y se los pasa a los periodistas que están aquí de guardia, inmediatamente nosotros calibramos y tenemos en cuenta el impacto social que tiene esa noticia", (Wilson Rodríguez, Coordinador de judiciales). 
"Lo más cercano a la realidad y lo más certero en los datos, es lo que uno trata de ser", (Andrés Gaitán, periodista).

"Lo primordial para un periodista judicial es hacerse a unas buenas fuentes, si tú tienes buenas fuentes créeme que no vas a tener limitantes a la hora de la reportería", (Harvey Jiménez, periodista).

"Que no aborde la cobertura enfocado solamente a una sola fuente, que sea lo más amplio posible, que sea pluri-fuentista", (Wilson Rodríguez, Coordinador de judiciales).

\section{DISCUSIÓN}

En cuanto a los hallazgos sobre cómo se establece la importancia de las noticias en el diario 'Al Día' se encontró que la portada funciona como una vitrina que permite exponer la mercancía noticiosa. Sin embargo, esto solo se da en un $75 \%$ de la muestra, es decir, que en el resto de las noticias estudiadas no existe esta exposición, lo cual podría deberse a que tuvieron poca relevancia a juicio del editor, del coordinador de judiciales o del jefe de redacción que son quienes toman las decisiones sobre el espacio dedicado a la noticia que están produciendo en la sala de redacción. Pese a ello aunque casi siempre la portada del diario contiene temas relacionados con el delito, a veces los resultados deportivos tienen relevancia a la hora de construir la portada. Según la muestra la noticia de homicidio no abre la sección en la mayoría de los casos lo cual representa una aparente contradicción con respecto al modo en que operan los diarios a nivel mundial, pues se supone que todas las noticias de homicidio importantes deberían abrir la sección dedicada a ellas, en este caso denominada: "Así fue", pero lo anterior tiene su explicación en el hecho de que el medio le da prelación a la pauta publicitaria, y por eso, solo en una tercera parte de los casos ocurre que este tipo de noticias abran la sección. El hallazgo de que la página en la que empieza la noticia sea par en más de la mitad de las noticias de la muestra permite suponer que el hecho de iniciar en página par o impar no tiene importancia para el medio, puesto que aunque en la mayoría de los casos las noticias de homicidio aparecen en una página par, el porcentaje de las impares está cercano en casi la mitad de los casos. Por otra parte, el tamaño de la nota periodística, que es otra manera de establecer la importancia de las noticias, está entre dos y tres cuartos de página: $34,6 \%$ y entre uno y dos cuartos de página, en un $20,8 \%$, lo cual podría deberse al hecho de que para el diario 'Al Día' el espacio ideal para las noticias de homicidio no es la página completa, como podría pensarse, sino menos de tres cuartos de página como lo revelan los hallazgos obtenidos en el análisis de contenidos. Es decir, que en la mayoría de la muestra el despliegue informativo, a lo sumo, alcanza los tres cuartos de página.

Dentro de esas dimensiones de la página hay que incluir el uso de elementos gráficos que acompañan a las noticias del delito, donde se encontró que, si bien están presentes en su gran mayoría, los esquemas o las infografías están ausentes y no suelen ser elementos que el diario usa para establecer la importancia de una noticia de este tipo.

En cuanto a la fotografía casi nunca el diario 'Al Día' prescinde de este recurso y suele usar más de una, lo cual supone que el diario le da un uso casi exclusivo a la 


\section{ANÁLISIS DE LA COBERTURA DEL HOMICIDIO, DESDE EL ENFOQUE DE PRODUCCIÓN NOTICIOSA, EN EL DIARIO "AL DÍA"}

fotografía y en cuanto a los recuadros están presentes en más de la mitad de las noticias de la muestra. En resumen, se confirma lo planteado por Aguillón (2008), para quien el diseño gráfico de los periódicos, que incluye la portada, el tamaño de la página y el uso de la fotografía y los recuadros es utilizado no solo para resaltarla noticia. En el caso de las fuentes solo el 17,1\% corresponde a las autoridades, lo cual supone que los periodistas arman la noticia de homicidio con los testimonios que recogen en el lugar de los hechos con familiares y vecinos de la víctima, esto permite al periodista acercarse más al suceso periodístico. Este proceso es duro para él, pues se enfrenta con frecuencia a la resistencia por parte de las autoridades y de los familiares, sobretodo de estos últimos quienes en el momento del duelo que viven, a veces, no están dispuestos a proporcionar información e incluso el reportero manifiesta que la crueldad del homicidio lo afecta.

El hallazgo de que en el diario 'Al Día' las autoridades no proporcionan la mayoría de la información con la que el reportero elabora o produce la noticiase contradice con lo que plantea Feinberg (2002), para quien los periodistas contribuyen a construir una imagen eficaz de las acciones de la policía, puesto que la información proporcionada por la autoridades se convierte en su fuente fundamental. Sin embargo, en el caso del diario 'Al Día', al momento de ir al lugar de los hechos y llevar a cabo el reporterismo, son los vecinos, amigos, familiares y testigos quienes proporcionan la materia prima con la cual el reportero trabaja. Por lo tanto, se puede afirmar que hay una baja presencia de las autoridades en las noticias de homicidio que se muestran en el diario 'Al Día'.

Además, en la instancia cualitativa se halló que el hecho de sangre o el muerto más reciente es un indicador para seleccionar la noticia, lo que confirma de acuerdo a Randall, Lee-Sammons y Hagner (1988), que los periodistas enfatizan la etapa más dramática y temprana del proceso judicial y que los medios están más interesados en la delincuencia común, que en otro tipo de crímenes, asimismo, que cada medio tiene una identidad en la manera en que presentan las noticias. Aunque el hallazgo de que la mayoría de las fuentes no provienen de las autoridades, sino en su mayoría de vecinos, amigos, familiares y testigos supone, según Aguillón (2010), que los periodistas privilegian sus voces informativas en cada uno de los textos que escriben. Lo que para Morales y Vallejo (2011), representa un abuso de las fuentes personales, según ellos usado para subrayar los efectos dramáticos de la información, que terminan configurando las actitudes profesionales de los periodistas. Estas actitudes, de acuerdo con Reese y Ballinger (2001), introducen un sesgo a la información con su subjetividad; sin embargo, el periodista de 'Al Día' trata de corregir esto con el uso de varias fuentes de información en el momento de la cobertura de la noticia, tal como señalaron en las entrevistas.

En la literatura académica autores como Tuchman (1973), Schudson (1989), Rodrigo (1989), Fontcuberta (1993) y Hernández (1997) se han enfocado en la producción o construcción de la noticia y los factores que intervienen en su elaboración. Otros como Canel y Sánchez-Aranda (1999) reconocen que tanto las actitudes profesionales como los elementos organizacionales son los factores que más influyen en el proceso de la producción noticiosa y en el caso del diario 'Al Día' la cobertura de noticias de homicidio es la materia prima. Esto no es nuevo, pues según Lundman (2003), los asesinatos, tienen una presencia o 'noticiabilidad' de 
vieja data en los medios, sin embargo, lo diferente que mostraron los resultados de la muestra estudiada es que existen criterios distintos de los que reconoce la literatura académica, que hacen parte de la rutina laboral del periodista y que según Frankenberg y Lozano-Rendón (2010) moldean e inciden en los contenidos de la producción noticiosa.

Los criterios de selección que los periodistas del Diario 'Al Día' usan al momento de producir la noticia, encontrados en la instancia cualitativa contienen tres elementos:

1. Impacto

2. Cercanía

3. Repercusión en la sociedad

Estos hallazgos nos muestran que en la producción noticiosa del Diario 'Al Día' los periodistas seleccionan la información teniendo en cuenta criterios orientados a romper la rutina del lector: construyen una noticia que lo sorprenda y le provoque interés, pero a la vez, tienen en cuenta también lo que haya sucedido en su barrio o en su entorno, para lograr la cercanía que quiere el medio. Cuando las noticias del delito cumplen estas condiciones todavía queda pendiente la repercusión que tengan en la sociedad y nada más impactante que la pérdida de una vida humana, producto de un hecho violento. Por ello, en la sección "Así fue" del diario 'Al Día', el hecho de sangre o el muerto más reciente es el indicador que más valora el periodista al momento de la selección noticiosa, lo cual a su vez genera que se cumplan los tres criterios usados por el periodista en la producción de la noticia y como tal los valores noticiosos ya mencionados de: impacto, cercanía y repercusión en la sociedad.

\section{CONCLUSIONES}

Este análisis concluye que para establecer la importancia de las noticias de homicidio el diario 'Al Día' usa elementos como: aparecer en la portada, abrir la sección y aparecer en página par o impar, sin embargo, estos no son usados con un criterio fijo, pues priman más las conveniencias económicas del medio.

En la cobertura de noticias de homicidios, al diario 'Al Día' le parece suficiente, en la mayoría de los casos, dedicarle tres cuartos de página a la cobertura noticiosa y prescindir de elementos gráficos como las infografías o esquemas. En cuanto a la producción noticiosa, prefieren más de una fotografía, y a veces, utilizan recuadros explicativos.

Las fuentes a las que más acude el periodista del diario 'Al Día' son familiares, testigos y vecinos, esto les permite lograr un acercamiento con el lector, aunque compromete la objetividad de la noticia, sin embargo, se acude a la práctica de conseguir el mayor número de fuentes posibles para reducir este sesgo, pese a ello la cobertura noticiosa no está exenta de dificultades para el periodista

Los valores noticiosos tradicionales se aplican también a este medio sensacionalista, pero existen otros como: impacto, cercanía y repercusión en la sociedad e incluso el hecho de sangre más reciente, que son los que en la práctica determinan la producción noticiosa. 


\section{REFERENCIAS}

Aguillón, P. (2010). Construcción ideológica de la violencia delictiva en la prensa venezolana. Comunicación y sociedad, 13, 69-100.

Canel, M. J., y Sánchez-Aranda, J. J. (1999). La influencia de las actitudes profesionales del periodista español en las noticias. In Anàlisi: Quaderns de comunicació i cultura (pp. 151-170).

Carpenter, S., Lacy, S. \& Fico, F. (2006). Network news coverage of high-profile crimes during 2004: a study of source use and reporter context. Journalism \& Mass Communication Quarterly, 83(4), 901-916.

Feinberg, S. L. (2002). Media effects: The influence of local newspaper coverage on municipal police size. American Journal of Criminal Justice, 26(2), 249-268.

Fontcuberta, Mar de (1993). La noticia. Pistas para percibir el mundo. España: Editorial Paidós.

Frankenberg, L., \& Lozano, J. C. (2010). Rutinas, valores y condicionantes en la producción de la noticia: el testimonio de cuatro directores de medios informativos en Monterrey, México/Routines, values and other influences in the production of news: The testimony of four managing editors in Monterrey, Mexico. Comunicación y sociedad, 23(1), 175. Disponible en: http://search.proquest.com/docview/1243095907?pq-origsite=gscholar

Hernández, M. (1997). La sociología de la producción de noticias. Hacia un nuevo campo de investigación en México. Comunicación y Sociedad. Universidad de Guadalajara, 30, 209-242. Disponible en:

www.publicaciones.cucsh.udg.mx/pperiod/comsoc/pdf/30 1997/209-242.pdf

Igartúa, J. (2006). Métodos cuantitativos de investigación en comunicación. España: Editorial Bosch.

Kayser, J. (1982). El diario francés. Fondation Nationale Des Sciences Politiques, París. A.T.E. Ronda General Mitr, 90. Barcelona, España.

Lance, W., Gressett, L. A. \& Haltom, W. (1985).Repairing the news: A case study of the news paradigm.Journal of Communication, 35(2), 50-68.

Lasorsa, D. \& Jia, D. (2007).When news reporters deceive: The production of stereotypes.Journalism \& Mass Communication Quarterly, 84(2), 281-298.

Lundman, R. J. (2003, September). The newsworthiness and selection bias in news about murder: Comparative and relative effects of novelty and race and gender typifications on newspaper coverage of homicide. In Sociological Forum, 18(3), pp. 357-386). Kluwer Academic Publishers-Plenum Publishers.

Marchetti, D. (2008). El análisis sociológico de la producción de información mediática. Comunicación y medios, 17(18). 
Morales, M., y Vallejo, M. (2011). Rutinas periodísticas y autopercepciones de los periodistas judiciales de los medios bogotanos. Signo y pensamiento, 31(59), 210-232.

Muñiz, C. (2007). Encuadres noticiosos e inmigración: del análisis de los contenidos al estudio de los efectos mediáticos. Tesis doctoral. Universidad de Salamanca.

Randall, D. M., Lee-Sammons, L. y Hagner, P. R. (1988). Common versus elite crime coverage in network news.Social Science Quarterly (University Of Texas Press), 69(4), 910-929.

Reese, S. D. y Ballinger, J. (2001). The roots of sociology of news: Remembering Mr. Gates and social control in the newsroom. Journalism \& Mass Communication Quarterly, 78(4), 641-658.

Rodrigo, M. (1989). La construcción de la noticia. España: Editorial Paidós.

Schudson, M. (1989). The sociology of news production. Media, culture and society, 11(3), 263-282.

Tuchman, G. (1973). Making news by doing work: Routinizing the unexpected.American Journal of Sociology, 110-131. 